\title{
AN OPERATOR FORMULATION OF THE MULTISCALE FINITE-VOLUME METHOD WITH CORRECTION FUNCTION*
}

\author{
IVAN LUNATI ${ }^{\dagger}$ AND SEONG H. LEE
}

\begin{abstract}
The multiscale finite-volume (MSFV) method has been derived to efficiently solve large problems with spatially varying coefficients. The fine-scale problem is subdivided into local problems that can be solved separately and are coupled by a global problem. This algorithm, in consequence, shares some characteristics with two-level domain decomposition (DD) methods. However, the MSFV algorithm is different in that it incorporates a flux reconstruction step, which delivers a fine-scale mass conservative flux field without the need for iterating. This is achieved by the use of two overlapping coarse grids. The recently introduced correction function allows for a consistent handling of source terms, which makes the MSFV method a flexible algorithm that is applicable to a wide spectrum of problems. It is demonstrated that the MSFV operator, used to compute an approximate pressure solution, can be equivalently constructed by writing the Schur complement with a tangential approximation of a single-cell overlapping grid and incorporation of appropriate coarse-scale mass-balance equations.
\end{abstract}

Key words. multiscale finite-volume method, domain decomposition, single-cell overlap, multiscale methods, multiphase flow in porous media, reservoir simulations

AMS subject classifications. 65N08, 65N55, 76M12, 76S05

DOI. $10.1137 / 080742117$

1. Introduction. In many branches of science and engineering, large elliptic or parabolic problems with highly heterogeneous coefficients need to be solved to describe the dynamics of physical systems. Geological porous media, such as aquifers and hydrocarbon reservoirs, are particularly challenging due to the hierarchy of scales involved, ranging from the pore scale (typically microns to millimeters) to the formation scale (kilometers) [9]. We generally assume that the macroscopic behavior is well described by Darcy's law (i.e., the pore-scale processes can be described in terms of average quantities defined on representative elementary volume [7]). It is, nevertheless, difficult to integrate all heterogeneity scales into numerical flow and transport models. For these reasons, an abundant literature has proposed upscaling techniques to reduce the number of degrees of freedom (see $[30,25]$ for a review).

In recent years, several multiscale methods have been developed to model multiphase flow and transport in geological formations. The main goal is to improve the description of multiphase systems by retaining information on the small-scale heterogeneity of medium properties and phase distribution, which are important due to the nonlinear nature of the partial differential equations involved [6]. To accomplish this, coupled local and global problems are solved numerically. In reservoir modeling, three major families of methods have been introduced: (1) The multiscale finiteelement method [11], which results in a flux field that is not conservative in general and poses difficulties for modeling transport; (2) The mixed multiscale finite-element method $[1,2,3,4,5,8]$, which is conservative but involves more degrees of freedom;

${ }^{*}$ Received by the editors November 27, 2008; accepted for publication (in revised form) September 1, 2009; published electronically October 22, 2009. This project was supported by Chevron ETC. http://www.siam.org/journals/mms/8-1/74211.html

${ }^{\dagger}$ Institute of Geophysics, University of Lausanne, Amphipole - UNIL Sorge, 1015 Lausanne, Switzerland (ivan.lunati@unil.ch).

${ }^{\ddagger}$ Chevron Energy Technology Co., 6001 Bollinger Canyon Rd., San Ramon, CA 94583 (seon@ chevron.com). 
and (3) the multiscale finite-volume (MSFV) method [12, 13, 14, 15, 16, 17, 20, 21], which provides a locally conservative flux field with the same number of degrees of freedom for the global problem as the multiscale finite-element method.

These techniques, which mainly deal with the elliptic (or parabolic) pressure equation, are closely related to upscaling methods in that approximate, mass-conservative solutions are sought rather than the exact answer. However, they obviously share some characteristics with domain decomposition (DD) methods $[24,28,26]$ and multigrid techniques [29], which have been developed to obtain efficient linear solvers for large problems. Recently, Nordbotten and Bjørstad [23] addressed and discussed the similarities between DD and MSFV. However, their analysis does not include in MSFV formulation the correction functions that have been introduced to deal with source terms, which can arise in the presence of capillarity or gravity effects $[19,21,16]$, and complex wells [31, 15].

In this paper we briefly review the general formulation of the MSFV method with correction function for rigorous treatment of source terms, which has been derived by Lunati and Jenny [18] (section 2); then we present an operator formulation of the MSFV with correction function, which is based on a classical reordering of unknowns and equations (section 3); finally, we discuss the similarities and outline the differences between the MSFV and DD methods and show that the MSFV methods are free of the drawbacks described in [23] (section 4).

2. Multiscale finite-volume method with correction function. In single or multiphase flow, a pressure equation is given in an elliptic or parabolic form. Here, we focus on the former case (incompressible phases) and refer the reader to $[17,18,32]$ for parabolic problems. Thus, we consider the inhomogeneous elliptic equation of the form

$$
\nabla \cdot \boldsymbol{v}=-\nabla \cdot(\mathbf{K} \nabla p-\boldsymbol{h})=q,
$$

where $p$ is the (unknown) pressure; $\mathbf{K}$ is a positive defined coefficient matrix; $\boldsymbol{h}$ is a vector that describes the effects of nonviscous forces, e.g., gravity or capillarity $[19,21] ; q$ is a source term per unit volume that can describe extended source terms and wells [17]; and $\boldsymbol{v}$ is the velocity vector.

Instead of solving (2.1) on the original fine grid, the MSFV method employs an auxiliary (primary) coarse grid, together with its dual (Figure 2.1). The dual coarse grid, which divides the domain $\Omega$ into a set of subdomains $\left\{\tilde{\Omega}^{d}\right\}_{d \in\left[1, N_{d}\right]}$, is used to define an approximate pressure solution. Referring to the two-dimensional (2D) case depicted in Figure 2.1, we observe that each edge is shared by two and each node by four adjacent duals if the grid is regular and Cartesian. The primary coarse grid, instead, defines a partition of the domain into cells, $\left\{\bar{\Omega}_{m}\right\}_{m \in\left[1, N_{n}\right]}$, that are centered on the nodes of the dual grid (we have, therefore, one coarse cell per each node of the dual grid). The coarse grid is used to define an approximate flux field that is conservative at the fine scale.

2.1. Pressure approximation. The approximate pressure is defined as a juxtaposition of local solutions computed in the dual cells:

$$
\tilde{p}=\bigcup_{d \in\left[1, N_{d}\right]} \tilde{p}^{d} \approx p
$$

The definition of the dual solutions, $\tilde{p}^{d}$, requires an appropriate localization assumption to assign the boundary conditions of the local problems. At this end, it is required 


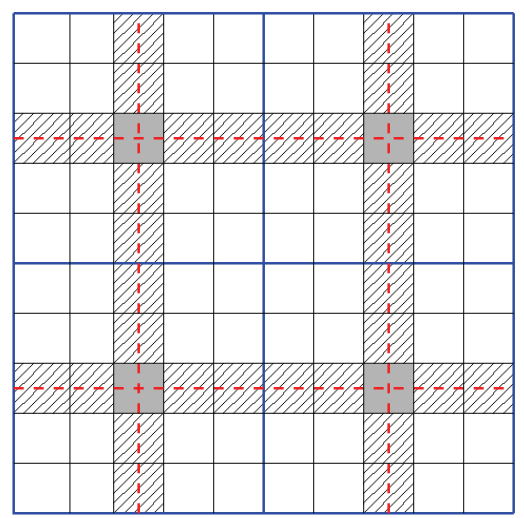

FIG. 2.1. Primary coarse grid (solid thick lines) and the dual coarse grid (dashed lines); the dual grid naturally defines a partition of the fine-mesh into internal (white cells), edge (shaded cells), and node points (grey cells) of the dual grid.

that the flux derivative in the direction perpendicular to the dual boundaries be zero, i.e.,

$$
\nabla \cdot\left[\left(\boldsymbol{\eta} \boldsymbol{\eta}^{T}\right) \boldsymbol{v}\right]=\left[\left(\boldsymbol{\eta} \boldsymbol{\eta}^{T}\right) \nabla\right] \cdot \boldsymbol{v}=\nabla_{\perp} \cdot \boldsymbol{v}=0,
$$

where $\boldsymbol{\eta} \boldsymbol{\eta}^{T}$ is the projector operator in the direction $\boldsymbol{\eta}$ perpendicular to edges of the duals, $\partial \tilde{\Omega}^{e}$. If the flow is divergence-free, $(2.3)$ is equivalent to requiring that $\tilde{p}^{d}$ is the solution of a reduced problem along the edges. Hence, the approximate pressure in a dual cell is solution of the problem

$$
\left\{\begin{array}{l}
\nabla \cdot \mathbf{K} \nabla \tilde{p}^{d}=r \quad \text { in } \quad \tilde{\Omega}^{d}, \\
\nabla_{\|} \cdot \mathbf{K} \nabla \tilde{p}^{d}=r_{\|} \\
\tilde{p}^{d}\left(\boldsymbol{x}_{i}\right)=p_{i},
\end{array}\right.
$$

where $p_{i}$ is the pressure at the node of the dual grid, $\boldsymbol{x}_{i}$, which is also called coarse-grid pressure; and we have defined $\nabla_{\|}=\left(\mathbf{I}-\boldsymbol{\eta} \boldsymbol{\eta}^{T}\right) \nabla, r=q+\nabla \cdot \boldsymbol{h}$, and $r_{\|}=q+\nabla_{\|} \cdot \boldsymbol{h}$.

The solution of (2.4) is then expressed as a linear combination of a set of basis functions, $\left\{\tilde{\varphi}_{j}^{d}\right\}$, independent of the node pressure, plus a correction function, $\tilde{\varphi}_{*}^{d}$, i.e.,

$$
\tilde{p}^{d}=\sum_{j} \tilde{\varphi}_{j}^{d} p_{j}+\tilde{\varphi}_{*}^{d} \quad \text { on } \quad \tilde{\Omega}^{d},
$$

and zero elsewhere. The linear combination of basis functions represents the solution of the localized homogeneous problem obtained by setting $r_{\|}=r=0$, whereas the correction function accounts for the effects of the right-hand side (r.h.s.) in (2.5) and describes all processes that do not scale with the coarse pressure. Since the homogeneous problem needs to be satisfied in $\tilde{\Omega}^{d}$ for any value of the coarse pressure, each basis function is obtained from the solution of

$$
\left\{\begin{array}{l}
\nabla \cdot \mathbf{K} \nabla \tilde{\varphi}_{j}^{d}=0 \quad \text { in } \tilde{\Omega}^{d}, \\
\nabla_{\|} \cdot \mathbf{K} \nabla \tilde{\varphi}_{j}^{d}=0 \\
\tilde{\varphi}_{j}^{d}\left(\boldsymbol{x}_{i}\right)=\delta_{i j} .
\end{array}\right.
$$

Each basis function $\tilde{\varphi}_{j}^{d}$ represents the contribution from a unit pressure signal at the node $\boldsymbol{x}_{j}$. For (2.5) to be the solution of (2.4), the correction function must be defined 
by the local problem:

$$
\left\{\begin{array}{l}
\nabla \cdot \mathbf{K} \nabla \tilde{\varphi}_{*}^{d}=r \quad \text { in } \quad \tilde{\Omega}^{d}, \\
\nabla_{\|} \cdot \mathbf{K} \nabla \tilde{\varphi}_{*}^{d}=r_{\|} \\
\tilde{\varphi}_{*}^{d}\left(\boldsymbol{x}_{i}\right)=0 .
\end{array}\right.
$$

To couple the local solutions in (2.4), the coarse-grid pressure coefficients, $p_{i}$, are determined by solving a set of coarse-scale mass conservation equations, which are obtained by integrating (2.1) over each coarse cell, $\bar{\Omega}_{i}$. Applying the Gauss (divergence) theorem, we get

$$
\int_{\bar{\Omega}_{i}} \nabla \cdot \mathbf{K} \nabla p d \boldsymbol{x}=\int_{\partial \bar{\Omega}_{i}} \mathbf{K} \nabla p \cdot \boldsymbol{\eta} d \Gamma=\int_{\bar{\Omega}_{i}} r d \boldsymbol{x}, \quad i \in\left[1, N_{n}\right],
$$

and using the approximate pressure, (2.5), we obtain the coarse-scale problem,

$$
\sum_{j \in \aleph_{i}} T_{i j}\left(p_{j}-p_{i}\right)=\sum_{d} \int_{\partial \bar{\Omega}_{i} \cap \tilde{\Omega}^{d}} \mathbf{K} \nabla \tilde{\varphi}_{*}^{d} \cdot \boldsymbol{\eta} d \Gamma-\int_{\bar{\Omega}_{i}} r d \boldsymbol{x}, \quad i \in\left[1, N_{n}\right],
$$

where $\aleph_{i}$ denotes the neighboring coarse cells of cell $i$ and the coarse-scale transmissibilities are defined by

$$
T_{i j}=-\sum_{d} \int_{\partial \bar{\Omega}_{i} \cap \tilde{\Omega}^{d}} \mathbf{K} \nabla \tilde{\varphi}_{j}^{d} \cdot \boldsymbol{\eta} d \Gamma .
$$

Equation (2.9) results in a 27-point stencil for three dimensions and a 9-point stencil for two dimensions. The coarse-scale operator, $T_{i j}$ does not include effects of $r$ and yields incorrect fluxes across $\partial \Omega_{i}$ for a given pressure drop between the nodes; the first term on the r.h.s. of (2.9) represents a correction to these inaccurate fluxes [19, 21].

The accuracy of the pressure approximation in (2.1) depends only on the quality of the localization assumption, (2.3). Given the localized problem to be satisfied and the basis-function definition, the correction function is uniquely defined.

2.2. Conservative flux approximation. The approximate pressure, $\tilde{p}$, satisfies the coarse-scale mass balance but yields fine-scale fluxes which are nonconservative at the dual boundaries. The approximate pressure is not the solution of (2.1) on the dual edges where transversal fluxes are neglected, (2.3). To avoid severe mass-balance errors when the flux field is used to model transport [12], a conservative flux approximation is constructed for the whole domain from a juxtaposition of local conservative pressure solutions computed in the volume defined by the primary partition, i.e.,

$$
\overline{\boldsymbol{v}}=-\mathbf{K} \nabla \bar{\psi}+\boldsymbol{h},
$$

where

$$
\bar{\psi}=\bigcup_{m \in\left[1, N_{n}\right]} \bar{\psi}_{m} .
$$

Each conservative local problem has the form of (2.1) and boundary conditions extracted from the approximate pressure solution, i.e.,

$$
\begin{cases}\nabla \cdot \mathbf{K} \nabla \psi_{i}=r & \text { in } \bar{\Omega}_{i}, \\ \nabla \bar{\psi}_{i} \cdot \boldsymbol{\nu}=\nabla \tilde{p} \cdot \boldsymbol{\nu} & \text { on } \partial \bar{\Omega}_{i},\end{cases}
$$

where $\boldsymbol{\nu}$ is the normal to the boundary. The resulting flux field is conservative everywhere by construction. 
3. Matrix formulation. Of course, a numerical implementation of the MSFV algorithm outlined in the previous section includes the discretization of the fine-scale problem. At this end we consider a finite-volume discretization of (2.1), which can be written in matrix form as

$$
A u=r,
$$

where the unknown variable (pressure) $u_{j}=u\left(x_{j}\right)$ is defined at a discrete set of points $\mathcal{I}_{f}=\left\{x_{j}\right\}_{j \in\left[1, N_{f}\right]}$. Hence we write $u=\left[u\left(x_{j} \in \mathcal{I}_{f}\right)\right]^{T}=\left[\begin{array}{llllll}u_{1} & u_{2} & \cdots & u_{j} & \cdots & u_{n}\end{array}\right]^{T}$ and similarly $r=\left[r\left(x_{j} \in \mathcal{I}_{f}\right)\right]^{T}$. The coefficient matrix $A=\left[a_{j k}\right]$ is symmetric and positive definite and hence nonsingular.

Here, we consider 2D problems with a 5-point stencil discretization, such that the resulting coefficient matrix, $A$, has pentadiagonal structure, if unknowns and equations are in lexicographic order. The directed graph associated with the 5-point stencil is illustrated in Figure 3.1(a). Referring to the starting point of the arrow as the "predecessor" and to the endpoint as the "successor," the direction of the arrow indicates that the predecessor contributes to the balance equation associated with the successor. Neglecting boundary points, we can say that the directed graph associated with $A$ is symmetric. The MSFV localization of the pressure problem is achieved by breaking the symmetry of the graph associated with the coefficient matrix.

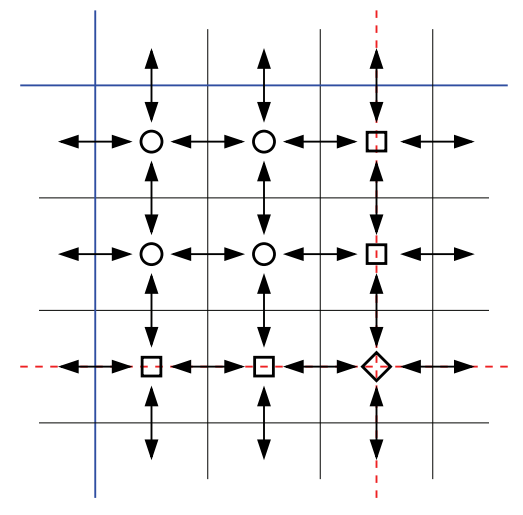

(a)

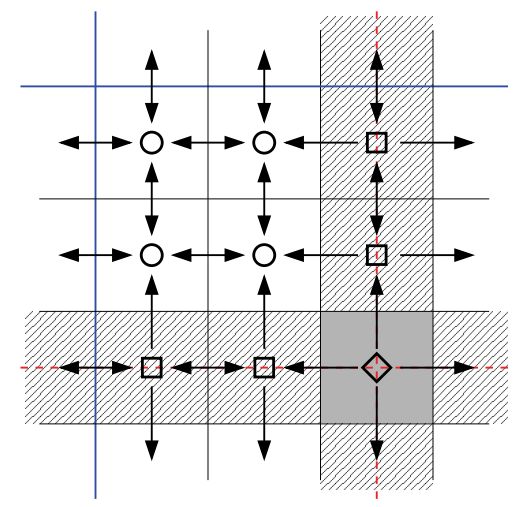

(b)

FIG. 3.1. Representation of the stencils used for the fine-scale solution (a) and for the multiscale solution with reduced-problem boundary conditions (b). Terming arrow starting points "predecessors" and endpoints "successors," an arrow indicates that the pressure value of the predecessor affects the mass balance of the successor.

3.1. Reordering. If the dual boundaries coincide with the segments connecting the fine-cell centers (Figure 2.1), then the dual mesh $\left\{\tilde{\Omega}^{d}\right\}_{d \in N_{d}}$ naturally defines a partition of the points $\left\{x_{j}\right\}$ into node, edge, and internal points (Figure 2.1), i.e.,

$$
\mathcal{I}_{f}=\mathcal{I}_{n} \cup \mathcal{I}_{e} \cup \mathcal{I}_{i}
$$

The sets $\mathcal{I}_{n}, \mathcal{I}_{e}$, and $\mathcal{I}_{i}$ consist of $N_{n}, N_{e}$, and $N_{i}$ points, respectively, and we have $N_{f}=N_{i}+N_{e}+N_{n}$. The localization rules expressed in (2.3) and (2.4) correspond to a graph in which the node points have no predecessor and internal points have only other internal points as successor (Figure 3.1(b)).

The graph of the MSFV operator can be easily described if the unknowns in (3.1) are reordered such that internal points appear first and node points last (see, 
e.g., [26] or [27] for an application related to reservoir simulations). Hence, we define the $N_{f} \times N_{f}$ permutation matrix $\tilde{P}$ associated to the dual grid, which reorders the unknown vectors such that

$$
\tilde{u}=\tilde{P} u=\left[\begin{array}{c}
\tilde{u}_{i} \\
\tilde{u}_{e} \\
\tilde{u}_{n}
\end{array}\right],
$$

where $\tilde{u}_{i}=\left[u\left(x_{j} \in \mathcal{I}_{i}\right)\right]^{T}, \tilde{u}_{e}=\left[u\left(x_{j} \in \mathcal{I}_{e}\right)\right]^{T}$, and $\tilde{u}_{n}=\left[u\left(x_{j} \in \mathcal{I}_{n}\right)\right]^{T}$. The permutation matrix has only one nonzero entry per row and column: if $\tilde{P}_{j k}=1$, then the element $u_{k}$ will become the element $\tilde{u}_{j}$ of the new vector. Recalling that permutation matrices are orthogonal, i.e., $\tilde{P}^{T}=\tilde{P}^{-1}$, we can rewrite (3.1) in the form

$$
\tilde{A} \tilde{u}=\tilde{r},
$$

where $\tilde{r}=\tilde{P} r$ and

$$
\tilde{A}=\tilde{P} A \tilde{P}^{T}=\left[\begin{array}{ccc}
\tilde{A}_{i i} & \tilde{A}_{i e} & 0 \\
\tilde{A}_{e i} & \tilde{A}_{e e} & \tilde{A}_{e n} \\
0 & \tilde{A}_{n e} & \tilde{A}_{n n}
\end{array}\right]
$$

The block $\tilde{A}_{j k}$ represents the effects of the unknowns $\tilde{u}_{k \in\{i, e, n\}}$ on the mass balance of the points $x \in \mathcal{I}_{j \in\{i, e, n\}}$. The diagonal blocks are block-diagonal. If properly ordered, the blocks of $\tilde{A}_{i i}$ and $\tilde{A}_{e e}$ are pentadiagonal and tridiagonal, respectively, whereas $\tilde{A}_{n n}$ is diagonal. $\tilde{A}_{i n}=\tilde{A}_{n i}^{T}=0$ because we are considering a 5-point stencil. The reordered matrix is connected to exactly the same symmetric directed graph as the original matrix.

3.2. MSFV matrix. Since we have simply reordered unknowns and equations, the problem in (3.4) is identical to the original linear system, (3.1). However, the MSFV method solves a different system, which we represent in the form

$$
M \tilde{u}=q,
$$

where we have defined

$$
M=\left[\begin{array}{ccc}
\tilde{A}_{i i} & \tilde{A}_{i e} & 0 \\
0 & M_{e e} & \tilde{A}_{e n} \\
0 & 0 & M_{n n}
\end{array}\right]
$$

and $q=\left[\begin{array}{lll}q_{i} & \tilde{r}_{e} & q_{n}\end{array}\right]^{T}$.

To understand (3.7), we recall that the block $\tilde{A}_{i e}$, respectively, $\tilde{A}_{e i}$, contains the active connections (internal points-edge points) that determine the pressure at the internal, respectively, edge, points. Satisfying (2.3) (or, equivalently, solving a reduced problem along the edges) requires that the "internal point-edge point" connections are removed when the edge-point equations are solved, i.e., $M_{e i}=0$. When solving for the internal points, however, connections with the edges are active, such that $M_{i e}=\tilde{A}_{i e} \neq 0$. This is illustrated by the oriented graph in Figure 3.1. Removing these connections also requires modifying the diagonal entries, such that $\tilde{A}_{e e}$ is substituted by

$$
M_{e e}=\tilde{A}_{e e}+\operatorname{diag}\left\{\sum_{i} \tilde{A}_{i e}^{T}\right\},
$$


where the operator "diag" transforms a vector into a diagonal square matrix. The diagonal block $\tilde{A}_{n n}$ has been replaced by a multidiagonal block $M_{n n}$, which is a 9diagonal matrix in the MSFV implementation. $M_{n n}$ is the coarse-scale operator and it is defined such that mass conservation on coarse control volumes is guaranteed (see (2.9)-(2.10)). Analogously, $\tilde{r}$ has been replaced by $q$. Note that $q_{i}=\tilde{r}_{i}$, whereas, in general, $q_{e} \neq \tilde{r}_{e}$ (for instance, in the presence of gravity); and we always have $q_{n} \neq \tilde{r}_{n}$. $M_{n n}$ and $q_{n}$ are derived in the next section; to simplify the exposition we consider the special case $q_{e}=\tilde{r}_{e}$. The sparsity patterns of the matrices $M$ and $A$ are compared in Figure 3.2.

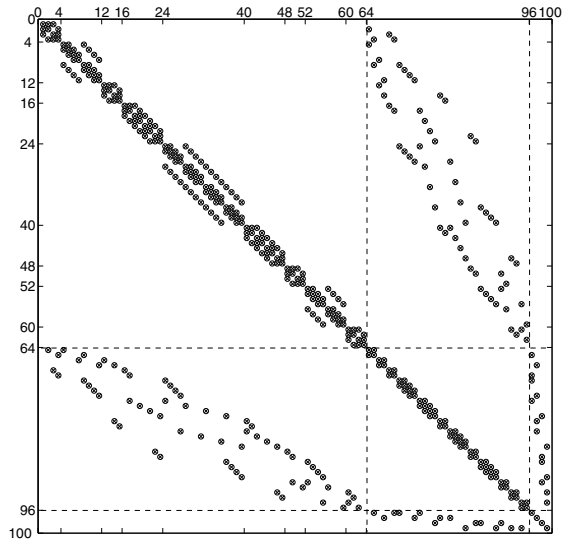

(a)

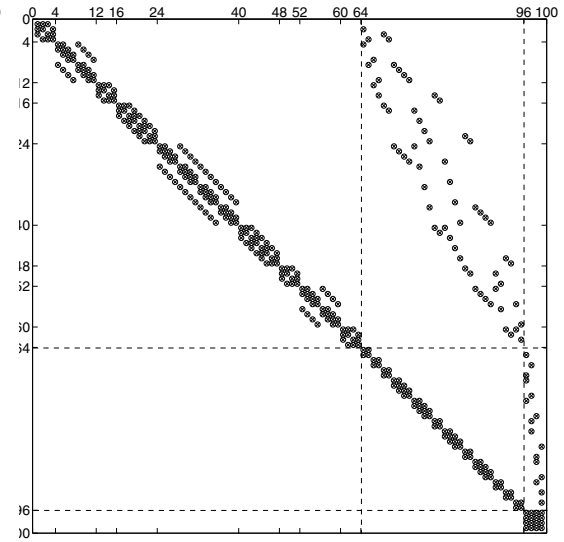

(b)

FIG. 3.2. Comparison of the sparsity patterns of the reordered fine-scale matrix (a) and the MSFV matrix (b) for the grid depicted in Figure 2.1. Localization is achieved by removing the internal point-edge point connections when the edge-point equations are solved; the fine-scale nodepoint equations are replaced by coarse-scale equations obtained by integrating over the control volumes defined by the coarse grid.

3.3. Coarse-scale problem. Since $M$ is block upper triangular, (3.7) can be readily solved by backward substitution, which yields

$$
\tilde{u}=B M_{n n}^{-1} q_{n}+C q,
$$

where we have defined the $N_{f} \times N_{n}$ prolongation operator,

$$
B=\left[\begin{array}{c}
\tilde{A}_{i i}^{-1} \tilde{A}_{i e} M_{e e}^{-1} \tilde{A}_{e n} \\
-M_{e e}^{-1} \tilde{A}_{e n} \\
I_{n n}
\end{array}\right]
$$

( $I_{n n}$ is the $N_{n} \times N_{n}$ identity matrix), and the $N_{f} \times N_{f}$ matrix,

$$
C=\left[\begin{array}{ccc}
\tilde{A}_{i i}^{-1} & -\tilde{A}_{i i}^{-1} \tilde{A}_{i e} M_{e e}^{-1} & 0 \\
0 & M_{e e}^{-1} & 0 \\
0 & 0 & 0
\end{array}\right] .
$$

(Note that $q_{n}$ does not contribute to $C q$ because the last column of $\mathrm{C}$ consists of zeros only; $q_{n}$ appears in (3.9) only through the solution of the coarse problem, (3.12) below.) 
This problem can be viewed as consisting of two steps: first, a coarse-scale problem is solved to compute the node (coarse-scale) pressures, i.e.,

$$
M_{n n} \tilde{u}_{n}=q_{n}
$$

then, the solution is prolonged on the fine grid,

$$
\tilde{u}=B \tilde{u}_{n}+C q .
$$

Using a restriction operator $R=\left[\begin{array}{lll}0 & 0 & I_{n n}\end{array}\right]$ the inverse multiscale matrix can be readily expressed as

$$
M^{-1}=B M_{n n}^{-1} R+C .
$$

Note that $\tilde{A}$ and $M$ are associated with different graphs. This means that, in general (i.e., for dimension $D>1$ ), the prolonged solution will not coincide with the finescale solution even if exact coarse-node pressures are assigned. This is due to the approximation introduced by the localization. For $D=1$, instead, the solution of the MSFV method with correction function is exact.

To derive the MSFV coarse-scale operator we first define the control-volume summation operator $\chi$, which is represented by an $N_{n} \times N_{f}$ matrix. Each row of $\chi=\left[\chi_{j \in\left[1, N_{n}\right]}\right]=\left[\chi_{j k}\right]$ corresponds to an element $\bar{\Omega}_{n}$, and we have the definition

$$
\chi_{j k}=\left\{\begin{array}{cc}
1 & \text { if } \quad x_{k} \in \bar{\Omega}_{j}, \\
0 & \text { otherwise. }
\end{array}\right.
$$

When applied to a vector of size $N_{f}$, this operator returns a vector of size $N_{n}$, whose entries are the sum of the values assumed by the original vector in the corresponding coarse cells. $\chi$ is the discrete analogue of the control-volume integral operator $\int_{\bar{\Omega}_{j}} d \boldsymbol{x}$ employed in section 2. A coarse-scale problem that satisfies the coarse-scale mass balance can be obtained by substituting (3.13) into (3.6) and applying the operator $\chi$, which yields

$$
\chi \tilde{A} \tilde{u}=\chi \tilde{A} B \tilde{u}_{n}+\chi \tilde{A} C q=\chi \tilde{r},
$$

from which we deduce that the coarse-scale operator is

$$
M_{n n}=\chi \tilde{A} B
$$

and the coarse-scale r.h.s. is

$$
q_{n}=\chi \tilde{r}-\chi \tilde{A} C q .
$$

If we define the operator

$$
F=I-R^{T} R+R^{T} \chi=\left[\begin{array}{ccc}
I_{i i} & 0 & 0 \\
0 & I_{e e} & 0 \\
\chi_{n i} & \chi_{n e} & I_{n n}
\end{array}\right]
$$

(where we have used the property $\chi_{n n}=I_{n n}$ ), we can express the r.h.s. of (3.6) as

$$
q=\left(F-R^{T} \chi \tilde{A} C\right) \tilde{r} .
$$


3.4. Relationship of $B$ and $C$ with basis and correction functions. Let us define a base of the subspace $\mathcal{I}_{n} \subset \mathcal{I}_{f}$, i.e., $\left\{e^{j}\right\}=\left\{e^{j} \mid x_{j} \in \mathcal{I}_{n}\right.$ and $\left.e^{j}=\left[\delta_{j i}\right]^{T}\right\}$. Then we can write

$$
B e^{j}=\bigcup_{d} \tilde{\varphi}_{j}^{d}
$$

where it appears that the columns of $B$ are given by the juxtaposition of the basis functions relative to the node $\boldsymbol{x}_{j}$. Comparing (2.5) and (3.13), we immediately obtain

$$
C q=\bigcup_{d} \tilde{\varphi}_{\star}^{d}=\tilde{\varphi}_{\star},
$$

which shows that $C q$ is the juxtaposition of the correction functions, which has been introduced in [16, 19, 21] for gravity and capillarity; in [15] for complex wells; and in very general form in [18]. It is evident from (3.13) that $\tilde{\varphi}_{\star}$ is the solution of the inhomogeneous problem with $\tilde{u}_{n}=0$.

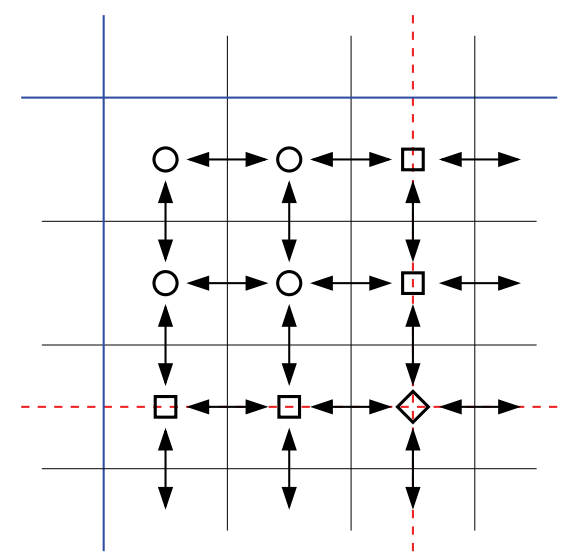

(a)

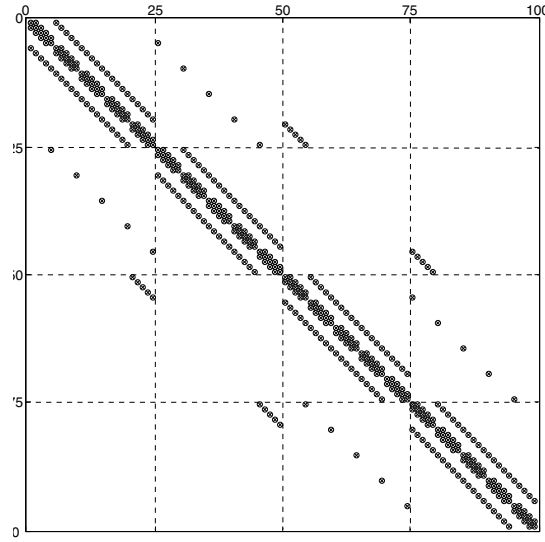

(b)

FIG. 3.3. (a) Representation of the stencils used for the construction of the conservative flux (the solid thick lines represent the boundaries of the primary coarse cells). An arrow from $A$ to $B$ indicates that the pressure value of $A$ affects the mass balance of $B$. (b) Sparsity pattern of the reordered coefficient matrix $\bar{A}=\bar{P} A \bar{P}^{T}$; the original pentadiagonal structure has been modified by the permutation $\bar{P}$ into a block-pentadiagonal structure.

3.5. The conservative flux field. To illustrate the discrete formulation of the conservative flux construction, it is useful to define the permutation operator $\bar{P}$ naturally associated with the coarse grid, $\left\{\bar{\Omega}_{n}\right\}_{n \in\left[1, N_{n}\right]}$. This permutation replaces the fine-scale lexicographic ordering by an ordering that is lexicographic in each coarse cell. The resulting matrix,

$$
\bar{A}=\bar{P} A \bar{P}^{T},
$$

has a pentadiagonal block structure if the coarse cells are also in lexicographic order (Figure 3.3(b)). Each diagonal block corresponds to a coarse cell $\bar{\Omega}_{i}$; off-diagonal blocks represent the mutual effect between adjacent blocks, i.e., they contain the transmissibilities between nodes belonging to two distinct but adjacent blocks. A conservative flux field is computed by solving local flow problems with Neumann 
boundary conditions, hence removing from the operator $\bar{A}$ the connections between points belonging to different coarse cells (Figure 3.3(a)). More rigorously, let $\bar{A}^{D}=$ $\operatorname{diag}(\bar{A})$, i.e., the block-diagonal part of the reordered matrix; then we define the block-diagonal matrix

$$
D=\bar{A}^{D}+\operatorname{diag}\left\{\sum_{j}\left(\bar{A}_{j k}-\bar{A}_{j k}^{D}\right)\right\} .
$$

The reordered fine-scale problem can be written in the form

$$
D \bar{u}+(\bar{A}-D) \bar{u}=\bar{r},
$$

where we have defined $\bar{u}=\bar{P} u$ and $\bar{r}=\bar{P} r$. In the MSFV algorithm, the second term on the left-hand side (l.h.s.) is approximate as $(\bar{A}-D) \bar{u} \approx(\bar{A}-D) \bar{P} \tilde{P}^{T} \tilde{u}$, where $\tilde{u}$ is the solution of $M \tilde{u}=\tilde{q}$, defined in the previous sections. Therefore, we write

$$
D \bar{u}_{c}=\bar{r}-(\bar{A}-D) \bar{P} \tilde{P}^{T} \tilde{u},
$$

where only fluxes across the boundaries of the primary grid contribute to the second term on the r.h.s.

4. Discussion. The matrix formulation presented in section 3 has been obtained following exactly the same steps outlined in section 2 for a continuum fine-scale problem. It is simply the fine-scale discrete form of the MSFV algorithm with correction function [18]. The matrix formulation, however, allows for a straightforward comparison with domain decomposition (DD) techniques. There are obvious similarities between the MSFV method (and multiscale methods in general) and classical DD preconditioners: they both employ a decomposition of the original problem into subdomains where local solutions are computed; also, the boundary conditions of the local problems expressed in (2.3) are identical to the tangential approximation of the DD literature.

There are, however, important characteristics of the MSFV method that differ from conventional DD. First, (approximate) acceptable solutions are obtained without iterating for most problems; second, the flux reconstruction step yields exact massconservative fine-scale fluxes even for approximate (inexact) pressure solution. The latter property is fundamental for applications in which phase or component transport are modeled. Mass conservation is enforced by construction employing primal and dual coarse grids: the primal coarse grid defines a set of coarse-scale control volumes and is used to define local problems to be solved to obtain an approximate flux field. Although employing two staggered grids is reminiscent of some overlapping DD techniques, the use is peculiar and allows computing a conservative flux field even if the pressure field is only an approximation of the exact fine-scale solution. The flux reconstruction step is a distinctive and inherent part of the MSFV algorithm and not a simple postprocessing operation. Another important property, closely related to the previous property, is that the MSFV flux approximation is conservative (at the fine scale) by construction, without the need for iterating. On the contrary, DD techniques are inherently iterative methods which deliver accurate solutions only after successive application of an operator (preconditioner). As such, mass conservation is guaranteed only within the numerical error at each iteration, and ultimately only for the converged solution. 
A DD preconditioner that allows a velocity reconstruction similar to that inherent to the MSFV method has been suggested by Nordbotten and Bjørstad [23], who enforce coarse-scale mass conservation by manipulating the original fine-scale system of equations. At this end, similarly to the MSFV algorithm, a second coarse grid is used to define a coarse-scale mass-balance equation, $\bar{\Omega}=\left\{\bar{\Omega}_{n}\right\}_{n \in\left[1, N_{n}\right]}$. Instead of directly considering (3.1), the equations relative to the nodes of the coarse grid, $x_{i \in\left[1, N_{n}\right]}$, are first replaced by the sum of all fine-scale equations relative to $x_{j} \in \bar{\Omega}_{i}$, which is a coarse-scale balance equation that guarantees mass conservation on the coarse grid (see (A.1)). Then the Schur complement with tangential approximation can be computed to localize the coarse-cell problems. As shown in the appendix, where we have developed these calculations in detail, this procedure yields a preconditioner identical to the MSFV operator (with correction function) as presented [18, 19, 21] and reformulated in terms of matrix operators in the present paper. Adopting the language of DD, we can say that the MSFV operator, if seen as a simple preconditioner, can be regarded as the Schur complement with tangential approximation of a single-cell overlapping DD method, provided that (3.1) has been replaced by the equivalent (A.1). Therefore, as demonstrated in the appendix, the algorithm suggested in [23] appears to be simply a reformulation of the MSFV algorithm with correction function [18].

The correction function allows consistent handling of the source term both at the fine scale and at the coarse scale. Hence, the MSFV algorithm is free of all drawbacks outlined in [23], which did not consider the recent development of the MSFV to treat source terms $[15,16,18,19,21,31]$. We stress that (2.3) uniquely defines the local problems, being fully equivalent to the tangential approximation for single-cell overlapping subdomains (i.e., edges are shared by adjacent duals). Although the localization assumption works reasonably well for a number of problems, it can be of interest to improve the accuracy of the MSFV method by performing iterations that minimize the flux inconsistency at the dual cell boundaries (see also [13]). At this end, the correction function, which allows a consistent treatment of the r.h.s., allows the construction of an iterative scheme converging to the exact fine-scale solution. Using the MSFV data structure, several iterative schemes can be developed [10, 22, 23].

Appendix. A Schur complement formulation satisfying coarse-scale mass balance can be obtained by introducing a balance equation for the coarse grid (see, e.g., [23]). The key idea is that, instead of writing the Schur complement of (3.1) directly, one considers the equation

$$
\tilde{A}^{\prime} \tilde{u}=F \tilde{r}
$$

where $F$ is given in (3.19) and we have defined

$$
\tilde{A}^{\prime}=F \tilde{A}=\left[\begin{array}{ccc}
\tilde{A}_{i i} & \tilde{A}_{i e} & 0 \\
\tilde{A}_{e i} & \tilde{A}_{e e} & \tilde{A}_{e n} \\
\tilde{A}_{n i}^{\prime} & \tilde{A}_{n e}^{\prime} & \tilde{A}_{n n}^{\prime}
\end{array}\right],
$$

with

$$
\begin{aligned}
& \tilde{A}_{n i}^{\prime}=\chi_{n i} \tilde{A}_{i i}+\chi_{n e} \tilde{A}_{e i}, \\
& \tilde{A}_{n e}^{\prime}=\chi_{n i} \tilde{A}_{i e}+\chi_{n e} \tilde{A}_{e e}+\tilde{A}_{n e} \\
& \tilde{A}_{n n}^{\prime}=\chi_{n e} \tilde{A}_{e n}+\tilde{A}_{n n} .
\end{aligned}
$$

The fine-scale equations relative to the nodes $x_{i \in\left[1, N_{n}\right]}$ have been replaced by a sum of all the equations relative to $x_{j} \in \bar{\Omega}_{i}$, which is a coarse-scale balance equation that 
guarantees mass conservation on the coarse grid $\bar{\Omega}=\left\{\bar{\Omega}_{n}\right\}_{n \in\left[1, N_{n}\right]}$. Obviously the resulting system is equivalent to (3.1).

By calculating the Schur complement of $\tilde{A}_{i i}^{\prime}=\tilde{A}_{i i}$, (A.1) becomes

$$
\left[\begin{array}{ccc}
\tilde{A}_{i i} & \tilde{A}_{i e} & 0 \\
0 & S_{e e} & \tilde{A}_{e n} \\
0 & S_{n e} & S_{n n}
\end{array}\right]\left[\begin{array}{c}
\tilde{u}_{i} \\
\tilde{u}_{e} \\
\tilde{u}_{n}
\end{array}\right]=\left[\begin{array}{ccc}
I_{i i} & 0 & 0 \\
-\tilde{A}_{e i} \tilde{A}_{i i}^{-1} & I_{e e} & 0 \\
-\tilde{A}_{n i}^{\prime} \tilde{A}_{i i}^{-1} & 0 & I_{n n}
\end{array}\right]\left[\begin{array}{c}
\tilde{r}_{i} \\
\tilde{r}_{e} \\
\chi \tilde{r}
\end{array}\right],
$$

where we have defined

$$
\begin{aligned}
S_{e e} & =\tilde{A}_{e e}-\tilde{A}_{e i} \tilde{A}_{i i}^{-1} \tilde{A}_{i e} \\
S_{n e} & =\tilde{A}_{n e}^{\prime}-\tilde{A}_{n i}^{\prime} \tilde{A}_{i i}^{-1} \tilde{A}_{i e}=\chi_{n e} S_{e e}+\tilde{A}_{n e} \\
S_{n n} & =\tilde{A}_{n n}^{\prime}
\end{aligned}
$$

Note that (A.6) is exact and fully equivalent to (3.1). By using the tangential component approximation, we write the second line of (A.6) as

$$
u_{e}=S_{e e}^{-1}\left(\tilde{r}_{e}-\tilde{A}_{e i} \tilde{A}_{i i}^{-1} r_{i}-\tilde{A}_{e n} \tilde{u}_{n}\right) \approx M_{e e}^{-1}\left(\tilde{r}_{e}-\tilde{A}_{e n} \tilde{u}_{n}\right)
$$

where we approximate $M_{e e} \approx S_{e e}$. Inserting (A.10) into the third line of (A.6) yields

$$
\left(S_{n n}-S_{n e} M_{e e}^{-1} \tilde{A}_{e n}\right) \tilde{u}_{n}=\chi \tilde{r}-\tilde{A}_{n i}^{\prime} \tilde{A}_{i i}^{-1} \tilde{r}_{i}-S_{n e} M_{e e}^{-1} \tilde{r}_{e}
$$

If we define

$$
M_{n n}^{\prime}=\left(S_{n n}-S_{n e} M_{e e}^{-1} \tilde{A}_{e n}\right)
$$

and

$$
q_{n}^{\prime}=\chi \tilde{r}-\tilde{A}_{n i}^{\prime} \tilde{A}_{i i}^{-1} \tilde{r}_{i}-S_{n e} M_{e e}^{-1} \tilde{r}_{e}
$$

we can write the tangential approximation of the Schur complement in the form

$$
\left[\begin{array}{ccc}
\tilde{A}_{i i} & \tilde{A}_{i e} & 0 \\
0 & M_{e e} & \tilde{A}_{e n} \\
0 & 0 & \hat{M}_{n n}^{\prime}
\end{array}\right]\left[\begin{array}{c}
\tilde{u}_{i} \\
\tilde{u}_{e} \\
\tilde{u}_{n}
\end{array}\right]=\left[\begin{array}{c}
\tilde{r}_{i} \\
\tilde{r}_{e} \\
q_{n}^{\prime}
\end{array}\right]
$$

This is in the same form of (3.6), which describes the MSFV operator. Further elaborating on (A.12) and (A.13), we obtain

$$
\begin{aligned}
\hat{M}_{n n}^{\prime} & =\tilde{A}_{n i}^{\prime} \tilde{A}_{i i}^{-1} \tilde{A}_{i e} M_{e e}^{-1} \tilde{A}_{e n}-\tilde{A}_{n e}^{\prime} M_{e e}^{-1} \tilde{A}_{e n}+\tilde{A}_{n n}^{\prime} \\
& =\chi\left(\left[\begin{array}{c}
\tilde{A}_{i i} \\
\tilde{A}_{e i} \\
0
\end{array}\right] \tilde{A}_{i i}^{-1} \tilde{A}_{i e} M_{e e}^{-1} \tilde{A}_{e n}-\left[\begin{array}{c}
\tilde{A}_{i e} \\
\tilde{A}_{e e} \\
\tilde{A}_{n e}
\end{array}\right] M_{e e}^{-1} \tilde{A}_{e n}+\left[\begin{array}{c}
0 \\
\tilde{A}_{e n} \\
\tilde{A}_{n n}
\end{array}\right] I_{n n}\right) \\
& =\chi \tilde{A} B=M_{n n}
\end{aligned}
$$

Copyright $@$ ( ) by SIAM. Unauthorized reproduction of this article is prohibited. 
and

$$
\begin{aligned}
& q_{n}^{\prime}=\chi \tilde{r}-\tilde{A}_{n i}^{\prime} \tilde{A}_{i i}^{-1}\left(\tilde{r}_{i}-\tilde{A}_{i e} M_{e e}^{-1} \tilde{r}_{e}\right)-\tilde{A}_{n e}^{\prime} M_{e e}^{-1} \tilde{r}_{e} \\
& =\chi\left(\tilde{r}-\left[\begin{array}{c}
\tilde{A}_{i i} \\
\tilde{A}_{e i} \\
0
\end{array}\right] \tilde{A}_{i i}^{-1}\left(\tilde{r}_{i}-\tilde{A}_{i e} M_{e e}^{-1} \tilde{r}_{e}\right)-\left[\begin{array}{c}
\tilde{A}_{i e} \\
\tilde{A}_{e e} \\
\tilde{A}_{n e}
\end{array}\right] M_{e e}^{-1} \tilde{r}_{e}\right) \\
& =\chi\left(\tilde{r}-\tilde{A}\left[\begin{array}{c}
\tilde{A}_{i i}^{-1}\left(\tilde{r}_{i}-\tilde{A}_{i e} M_{e e}^{-1} \tilde{r}_{e}\right) \\
M_{e e}^{-1} \tilde{r}_{e} \\
0
\end{array}\right]\right)=\chi(\tilde{r}-\tilde{A} C q)=q_{n},
\end{aligned}
$$

respectively. This proves that the matrix used to compute the dual pressure in the MSFV method $[18,21]$ is identical to the matrix obtained by computing the Schur complement with tangential approximation of the fine-scale problem, provided that the fine-scale nodal equations are replaced by coarse-scale balance equations.

Acknowledgments. The authors wish to thank Hamdi Tchelepi, Brad Mallison, and Hui Zhou for many constructive discussions.

\section{REFERENCES}

[1] J. E. AARNes, On the use of a mixed multiscale finite element method for greater flexibility and increased speed or improved accuracy in reservoir simulation, Multiscale Model. Simul., 2 (2004), pp. 421-439.

[2] J. E. Aarnes, V. Kippe, And K. A. Lie, Mixed multiscale finite elements and streamline methods for reservoir simulation of large geomodel, Adv. Water Res., 28 (2005), pp. 257271.

[3] T. Arbogast, Numerical Subgrid Upscaling of Two Phase Flow in Porous Media, Technical report, Texas Institute for Computational and Applied Mathematics, The University of Texas at Austin, Austin, TX, 1999.

[4] T. Arbogast, Implementation of a locally conservative numerical subgrid upscaling scheme for two phase Darcy flow, Comput. Geosci., 6 (2002), pp. 453-481.

[5] T. Arbogast and S. L. Bryant, Numerical Subgrid Upscaling for Waterflood Simulations, presented at the SPE Symposium on Reservoir Simulation, Houston, TX, 2001, SPE 66375.

[6] K. Aziz And A. Settari, Petroleum Reservoir Simulation, Applied Science Publ., London, 1979.

[7] J. Bear and Y. Bachmat, Introduction to Modeling of Transport Phenomena in Porous Media, Kluwer Academic Publishers, Dordrecht, The Netherlands, 1990.

[8] Z. Chen And T. Y. Hou, A mixed finite element method for elliptic problems with rapidly oscillating coefficients, Math. Comput., 72 (2003), pp. 541-576.

[9] J. H. Cushman, Dynamics of Fluids in Hierarchical Porous Media, Academic Press, New York, 1990.

[10] H. Hajibeygi, G. Bonfigli, M. A. Hesse, and P. Jenny, Iterative multiscale finite-volume method, J. Comput. Phys., 227 (2008), pp. 8604-8621.

[11] T. Y. Hou AND X. H. Wu, A multiscale finite element method for elliptic problems in composite materials and porous media, J. Comput. Phys., 134 (1997), pp. 169-189.

[12] P. Jenny, S. H. Lee, And H. TChelePI, Multi-scale finite-volume method for elliptic problems in subsurface flow simulation, J. Comput. Phys., 187 (2003), pp. 47-67.

[13] P. Jenny, S. H. Lee, And H. Tchelepi, Adaptive multiscale finite-volume method for multiphase flow and transport in porous media, Multiscale Model. Simul., 3 (2004), pp. 50-64.

[14] P. Jenny, S. H. Lee, And H. TChelepi, Adaptive fully implicit multi-scale finite-volume method for multi-phase flow and transport in heterogeneous porous media, J. Comput. Phys., 217 (2006), pp. 627-641.

[15] P. Jenny and I. Lunati, Modeling complex wells with the multi-scale finite-volume method, J. Comput. Phys., 228 (2009), pp. 687-702.

[16] S. H. Lee, C. Wolfsteiner, AND H. A. TChelePI, Multiscale finite-volume formulation for multiphase flow in porous media: Black oil formulation of compressible, three-phase flow with gravity, Comput. Geosci., 12 (2008), pp. 351-366.

Copyright $@$ by SIAM. Unauthorized reproduction of this article is prohibited. 
[17] I. Lunati And P. Jenny, Multi-scale finite-volume method for compressible flow in porous media, J. Comput. Phys., 216 (2006), pp. 616-636.

[18] I. Lunati And P. Jenny, The multiscale finite-volume method-a flexible tool to model physically complex flow in porous media, in Proceedings of the European Conference on Mathematics of Oil Recovery X, Amsterdam, The Netherlands, 2006, paper B010; available online from http://www.ifdmavt.ethz.ch/research/group_pj/publications/2006_LunatJ_Ecmor.pdf.

[19] I. LunAti AND P. JENNY, A multiscale finite-volume method for three-phase flow influenced by gravity, in Proceedings of the 16th International Conference on Computational Methods for Water Resources, Copenhagen, Denmark, 2006, paper 123; available online from http://proceedings.cmwr-xvi.org/materialDisplay.py? contribld=123\&amp;sessionld=11\&amp;materialld=paper\&amp; confld =a051.

[20] I. Lunati And P. Jenny, Treating highly anisotropic subsurface flow with the multiscale finitevolume method, Multiscale Model. Simul., 6 (2007), pp. 308-318.

[21] I. Lunati And P. Jenny, Multiscale finite-volume method for density-driven flow in porous media, Comput. Geosci., 12 (2008), pp. 337-350.

[22] I. Lunati, M. Tyagi, And S. H. Lee, An iterative multiscale finite volume algorithm converging to exact solution, J. Comput. Phys., submitted.

[23] J. M. Nordbotten And P. E. BJøRSTAd, On the relationship between the multiscale finitevolume method and domain decomposition preconditioners, Comput. Geosci., 12 (2008), pp. 367-376.

[24] A. Quarteroni and A. Valli, Domain Decomposition Methods for Partial Differential Equations, Oxford University Press, Oxford, UK, 1999.

[25] Ph. Renard and G. De Marsiliy, Calculating equivalent permeability: A review, Adv. Water Res., 20 (1997), pp. 253-278.

[26] B. Smith, P. Buørstad, And W. Gropp, Domain Decomposition: Parallel Multilevel Methods for Elliptic Partial Differential Equations, Cambridge University Press, Cambridge, UK, 2004.

[27] H. A. TChelepi And J. Wallis, Apparatus, Methods and System for Improved Reservoir Simulation Using an Algebraic Cascading Class Linear Solver, International Patent Classification E21B 49/00, International Publication Number WO2006/138530 A1, 2006.

[28] A. Toselli And O. Widlund, Domain Decomposition Methods: Algorithms and Theory, Springer, Heidelberg, 2004.

[29] U. Trottenberg, C. W. Oosterlee, and A. Schuller, Multigrid, Academic Press, London, 2001.

[30] X. H. Wen And J. J. Gómez-Hernández, Upscaling hydraulic conductivities in heterogeneous media: An overview, J. Hydr., 183 (1996), pp. R9-R32.

[31] C. Wolfsteiner, S. H. Lee, And H. A. TChelepi, Well modeling in the multiscale finite volume method for subsurface flow simulation, Multiscale Model. Simul., 5 (2006), pp. 900-917.

[32] H. Zhou AND H. A. TChelePI, Operator based multiscale method for compressible flow, in Proceeding of the SPE Syposium on Reservoir Simulation, Houston, TX, 2008, SPE 106254.

Copyright (c) by SIAM. Unauthorized reproduction of this article is prohibited. 